\title{
Practice Parameter: Diagnosis and prognosis of new onset Parkinson disease (an evidence-based review)
}

\section{Report of the Quality Standards Subcommittee of the American Academy of Neurology}

\author{
O. Suchowersky, MD; S. Reich, MD; J. Perlmutter, MD; T. Zesiewicz, MD; G. Gronseth, MD; \\ and W.J. Weiner, MD
}

\begin{abstract}
Objective: To define key issues in the diagnosis of Parkinson disease (PD), to define features influencing progression, and to make evidence-based recommendations. Two clinical questions were identified: 1) Which clinical features and diagnostic modalities distinguish PD from other parkinsonian syndromes? 2) Which clinical features predict rate of disease progression? Methods: Systematic review of the literature was completed. Articles were classified according to a four-tiered level of evidence scheme. Recommendations were based on the evidence. Results and Conclusions: 1. Early falls, poor response to levodopa, symmetry of motor manifestations, lack of tremor, and early autonomic dysfunction are probably useful in distinguishing other parkinsonian syndromes from Parkinson disease (PD). 2. Levodopa or apomorphine challenge and olfactory testing are probably useful in distinguishing PD from other parkinsonian syndromes. 3. Predictive factors for more rapid motor progression, nursing home placement, and shorter survival time include older age at onset of $\mathrm{PD}$, associated comorbidities, presentation with rigidity and bradykinesia, and decreased dopamine responsiveness. Future research into methods for earlier and more accurate diagnosis of the disease and identification and clarification of predictive factors of rapid disease progression is warranted.
\end{abstract}

NEUROLOGY 2006;66:968-975

Statement of purpose. The Quality Standards Subcommittee (QSS) develops scientifically sound, clinically relevant practice parameters to aid in the practice of neurology based on available evidence. This article addresses diagnostic and prognostic issues important for the management of Parkinson disease (PD). These recommendations are meant to

Additional material related to this article can be found on the Neurology Web site. Go to www.neurology.org and scroll down the Table of Contents for the April 11 issue to find the title link for this article. address the needs of specialists and nonspecialists caring for people with PD.

Background and justification. PD is a neurodegenerative disorder caused by a loss of dopaminergic neurons in the substantia nigra, as well as other dopaminergic and nondopaminergic areas of the brain. It has an estimated prevalence of up to 329/ $100,000 .{ }^{1}$ Although PD is common, it can be difficult to diagnose clinically, particularly in early stages, and approximately 5 to $10 \%$ of patients with PD are

\section{Editorial, see page 966 \\ See also pages 976,983 , and 996}

This article was previously published in electronic format as an Expedited E-Pub at www.neurology.org.

From the University of Calgary (O.S.), AB, Canada; University of Maryland Hospital (S.R.), Baltimore; Division of Radiation Sciences (J.P.), St. Louis, MO; Movement Disorder Center (T.Z.), Tampa, FL; University of Kansas (G.G.), Kansas City; and University of Maryland School of Medicine (W.J.W.), Baltimore. Quality Standards Subcommittee Members are listed in appendix E-5 on the Neurology Web site at www.neurology.org.

Approved by QSS July 30, 2005; Practice Committee December 15, 2005; Board of Directors February 23, 2006.

Endorsed by the National Parkinson Foundation and the Parkinson's Disease Foundation.

Disclosures are provided after the text.

Received August 16, 2005. Accepted in final form February 16, 2006.

Address correspondence and reprint requests to the American Academy of Neurology, 1080 Montreal Avenue, St. Paul, MN 55116.

968 Copyright () 2006 by AAN Enterprises, Inc. 
misdiagnosed. ${ }^{2}$ Conversely, up to $20 \%$ of patients diagnosed with PD reveal alternative diagnoses at autopsy, such as multiple system atrophy (MSA), progressive supranuclear palsy (PSP), AD-type pathology, and cerebrovascular disease..$^{2-6}$ Based on these studies, diagnostic criteria have been developed, with the most commonly used being the UK PD Society Brain Bank criteria ${ }^{2}$ (see appendix E-1 on the Neurology Web site at www.neurology.org). However, it has been suggested that an accuracy of $90 \%$ is the best that can be achieved with clinical assessment and clinical diagnostic criteria. ${ }^{4}$

Although symptomatic therapy can provide benefit for many years, PD is a progressive disorder that will eventually result in significant morbidity. Knowledge of the features that predict the rate of progression would empower clinicians to better counsel patients regarding prognosis and life expectancy. Improvement in diagnostic accuracy and the ability to predict the rate of progression would also impact on the ability to assess neuroprotective therapies that may delay the progression of the disease (see Practice Parameter on neuroprotective strategies).

Clinical question statement. This practice parameter addresses the following two clinically relevant questions regarding the diagnosis and prognosis of PD: 1 . Which clinical features and diagnostic modalities distinguish PD from other parkinsonian syndromes? 2. Which clinical features predict rate of disease progression?

Description of the analytical process. The QSS of the American Academy of Neurology identified five movement disorder specialists and a general neurologist with methodologic expertise. For the literature review, the following databases were searched: MEDLINE, EMBASE, CINHAL, and Cochrane Database of Systematic Reviews for the years 1997 to 2002. Only articles written in English were included. A second MEDLINE search covered 1966 through August 2004, followed by another search using the bibliographies of retrieved articles and knowledge from the expert panel extending to January 2005. At least two panel members reviewed each article. If a panelist was an author of one of the articles, at least two other panelists reviewed that article. If a disagreement was identified, consensus was reached by discussion with the whole group. The risk of bias for each study was determined using the classification of evidence scheme in appendix E-2. The strength of the practice recommendation was linked directly to the level of evidence (appendix E-3). Conflicts of interest were disclosed. Support was provided by the American Academy of Neurology. Writing meetings were funded by the Michael J. Fox Foundation. Panelists were not compensated.

Results, key words, and inclusion/exclusion criteria. For question 1: Search terms: Parkinson disease, neurologic examination, clinical characteristics, neuroimaging, radionuclide imaging, ultrasonography, differential diagnosis, autopsy, SPECT, PET, (levo- dopa or dopamine or apomorphine) challenge, olfactory. The search resulted in 176 articles. Inclusion criteria: At least 10 subjects with PD and 10 in the comparison group. Categories found: clinical, acute challenge testing, radiologic evaluation, neurophysiologic testing, biochemical testing, CSF examination, olfactory testing. Data presented in sufficient detail to allow calculation of sensitivities and specificities.

Results: Of the original 176 articles identified, 48 were found to be unrelated to the topic or were review articles. A total of 128 articles were reviewed; 31 articles satisfied inclusion criteria.

For question 2: Search terms: Parkinson disease, disease progression, muscle rigidity, tremor, hypokinesia, equilibrium, posture, gait. The search resulted in 59 articles. Inclusion criteria: Longitudinal data to assess putative factors, with an outcome measure that included motor progression measured by a validated rating scale, motor fluctuations, dementia, quality of life, and death. Articles were excluded if published before 1990 because of changes in the case definition of PD.

Results: Of the original 59 articles, 32 were not related to the topic or were review articles. Twentyseven articles were reviewed, and seven fulfilled inclusion criteria.

\section{Analysis of the evidence.}

Question 1: Which clinical features and diagnostic rodalities distinguish PD from other parkinsonian syndromes? We identified four articles that addressed the diagnostic accuracy of clinical features that were helpful in differentiating PD from other forms of parkinsonism (table E-1). ${ }^{7-10}$ A Class II case control study of 77 patients with pathologic diagnoses of different parkinsonian conditions including corticobasal degeneration (CBD), dementia with Lewy bodies (DLB), MSA, PD, and PSP revealed that falling within 1 year of diagnosis was a strong predictor of other forms of parkinsonism. ${ }^{9}$ Recurrent falling within the first year was a strong predictor of PSP, whereas time to onset of falling was more delayed in CBD, DLB, and MSA and most prolonged in PD.

A Class II retrospective study of 100 autopsyconfirmed cases of PD and 38 with $\mathrm{MSA}^{10}$ used a multivariate logistic regression analysis to construct a model of clinical features which help to distinguish PD from MSA. Based on features present until death, and assigning point values to each, the following variables yielded the best prediction: poor response to levodopa (two points); autonomic dysfunction, consisting of symptomatic postural hypotension, urinary urge incontinence, fecal incontinence, urinary retention requiring catheterization, and persistent erectile dysfunction (two points); speech or bulbar dysfunction (two points); absence of levodopa induced confusion (four points); and falls (four points). A point score of $\geq 11$ yielded a sensitivity of $90.3 \%$ and specificity of $92.6 \%$ in predicting patients had MSA rather than PD. It is perhaps 
more important to make a distinction between PD and MSA earlier in the disease course. In that setting, within the first 5 years, the following variables and point values were most predictive: poor response to levodopa (two points), early motor fluctuations (two points), autonomic dysfunction (two points), and rigidity (two points). A score of $\geq 4$ had a sensitivity of $87.1 \%$ and specificity of $70.5 \%$ of predicting MSA.

A Class II retrospective cohort study of 800 patients diagnosed with PD by movement disorder specialists and enrolled in DATATOP found that 65 individuals $(8.1 \%)$ were ultimately determined to have an alternative diagnosis. ${ }^{8}$ Clinical features that distinguished the two groups at baseline included higher Hoehn and Yahr stage, higher Unified PD Rating Scale (UPDRS) scores for bradykinesia, postural instability and gait difficulty, and a lower tremor score in the group with other forms of parkinsonism.

In a Class III case control study of 20 people with PD and 32 with either PSP or MSA identified pathologically, only $5 \%$ of patients with PD had orthostatic hypotension and all cases of PD were levodopa responsive. ${ }^{7}$ Lack of tremor, symmetry, and rapid progression were more likely to be associated with PSP or MSA, rather than PD.

Drug challenge: Response to levodopa or apomorphine. As the response to chronic levodopa therapy is an important factor in distinguishing PD from a parkinsonian syndrome, it follows that an acute dopaminergic challenge with either levodopa or apomorphine may have similar predictive value, Several meta-analyses of levodopa and apomorphine challenge tests have been published. ${ }^{11-13} \mathrm{~A}$ review of studies of either levodopa or apomorphine challenge tests yielded two articles that met our inclusion criteria.

A Class I double-blind study of 82 patients presenting with parkinsonian symptoms were given an acute levodopa challenge of 250/50 mg of levodopacarbidopa orally (table E-2). ${ }^{14}$ A blinded rater assessed the UPDRS; improvement of $30 \%$ or greater was felt to be positive and supportive of a diagnosis of PD. At 24 months, the patients were retested and the diagnosis of $\mathrm{PD}$ made clinically in 55 of the patients, based on the UK Parkinson Brain Bank criteria $^{6}$ (appendix E-1). The test had a sensitivity of $70.9 \%$ and a specificity of $81.4 \%$ for predicting the eventual diagnosis of PD. ${ }^{14}$ When the patients were divided into three groups based on UPDRS scores, the sensitivity for those with scores $\leq 10$ was similar to the entire group (71.4\%), but the specificity increased to $100 \%$. In those patients with more advanced signs at presentation (UPDRS $\geq 21$ ), the sensitivity dropped to $36.4 \%$ and the specificity was $87 \%$.

In a Class II cohort survey of a levodopa and apomorphine challenge to 134 consecutive patients with parkinsonism, the response to a single oral dose of levodopa $(250 \mathrm{mg})$ had a sensitivity of $77.1 \%$ and a specificity of $71.1 \%$ for distinguishing PD from other forms of parkinsonism (table E-2). ${ }^{15}$ Comparable val- ues were observed with varying doses of apomorphine (1.5 to $4.5 \mathrm{mg}$ ) given subcutaneously. Results ranged from $65.9 \%$ sensitivity and $70.5 \%$ specificity with a $1.5 \mathrm{mg}$ dose, to $66.7 \%$ sensitivity and $76.5 \%$ specificity with a $4.5 \mathrm{mg}$ dose. The specific diagnosis (PD, PSP, MSA, or parkinsonism not otherwise specified) was based almost exclusively on clinical rather than pathologic criteria.

It should be noted that patients in both studies were pretreated with domperidone 2 to 3 days prior to administration of the challenge to prevent peripheral dopaminergic side effects (nausea, emesis, and hypotension).

Drug challenge: Stimulation of growth hormone $(\mathrm{GH})$ with clonidine. The ability of clonidine, a centrally acting $\alpha 2$ adrenergic receptor agonist, to stimulate the release of $\mathrm{GH}$ has been proposed as a method to distinguish PD from MSA. Of the studies reviewed, only one met inclusion criteria. In this Class II study, 32 patients with early parkinsonism were followed for 2 years, until a diagnosis could be made using clinical criteria and IBZM-SPECT. Of the 21 patients with PD and 11 with MSA, the frequency of positive and negative responses did not differ between the two groups. ${ }^{16}$

Olfaction. Olfaction is frequently impaired in $\mathrm{PD}$, suggesting smell may be a potentially useful test to distinguish PD from related disorders. ${ }^{17}$ Three Class II studies were identified that met inclusion criteria. In a controlled but unblinded study using the University of Pennsylvania Smell Identification Test (UPSIT), patients with PSP $(\mathrm{n}=21)$ had similar olfaction to normal controls $(\mathrm{n}=21)$, but differed from patients with PD $(\mathrm{n}=21)(p<0.001) .{ }^{18}$ In a prospective study of 50 patients with parkinsonism evaluated with "Sniffin' Sticks," all 37 patients determined to have PD had moderate to severe hyposmia or anosmia, while the 13 patients with other causes of parkinsonism (MSA, PSP) and essential tremor (ET) had normal or only mild to moderate hyposmia. ${ }^{19}$ Wenning et al. ${ }^{20}$ compared olfaction in patients with PD $(\mathrm{n}=118), \operatorname{MSA}(\mathrm{n}=29), \operatorname{PSP}(\mathrm{n}=$ $15), \operatorname{CBD}(\mathrm{n}=7)$, and normal controls $(\mathrm{n}=123)$ using the UPSIT. Patients with PD scored significantly worse than all other patient groups, as well as controls. Patients with PSP and CBD achieved normal values. Patients with MSA scored worse than controls, but significantly better than PD. A cutoff score of 25 (out of possible 40 ) yielded a sensitivity of $77 \%$ and specificity of $85 \%$ in distinguishing PD from other parkinsonian syndromes.

Diagnostic neurophysiologic testing. A variety of noninvasive or minimally invasive procedures have been proposed to distinguish PD from parkinsonian syndromes. Twelve Class III articles ${ }^{21-31}$ were identified (table E-3). In these studies, diagnosis of PD or other parkinsonian syndromes were based on clinical criteria.

In one Class III study, electrooculography was used to determine the frequency of square wave jerks in 118 patients with PD, PSP, and MSA (table 
E-3). ${ }^{27}$ Using a cutoff of $>10$ square wave jerks/ minute as indicative of impaired gaze holding, positive results were observed in $7 / 8(87.5 \%)$ patients with PSP, 16/25 (64\%) with MSA, but only 13/85 (15.3\%) with PD. These results suggest that frequent square wave jerks in this population may assist in the diagnosis of PSP or MSA. Vidailhet et al. ${ }^{30}$ performed eye movement recordings in 14 patients with PD, 14 with MSA, 10 with CBD, and 10 with PSP (table E-3). Latency of horizontal saccades was significantly slower in CBD than PD and other parkinsonian syndromes. Horizontal saccade gain was significantly slower in PSP than PD and other parkinsonian syndromes. The percentage of errors on the anti-saccade task was significantly greater in PSP compared to PD and MSA, but not CBD. Square wave jerks were present as follows: PD 18\%, MSA $7 \%$, CBD 20\%, and PSP $60 \%$.

Six Class III studies of autonomic testing in parkinsonism met inclusion criteria. One study compared urodynamics in 21 patients with $\mathrm{PD}$ and 27 with MSA. ${ }^{28}$ All of the MSA patients had abnormal results, compared to $13 / 21$ (61.9\%) of patients with PD.

Sympathetic skin responses (SSR) and R-R interval variability (RRIV) were compared in $26 \mathrm{PD}$ and MSA patients. ${ }^{21}$ Nine of $13(69.2 \%)$ MSA patients had an abnormal SSR compared to only 1/13 (7.7\%) PD patients for a sensitivity of $69 \%$ and a specificity of $92 \%$. RRIV after deep breathing was abnormal in 8/9 (89\%) patients with MSA compared to only 3/11 $(27.3 \%)$ patients with PD $(p=0.02)$.

In another study of autonomic function, heart rate variability (HRV) was reduced during forced respiration in patients with MSA, but not PD or PSP as compared to controls $(p<0.01) .{ }^{24}$ However, pathologic HRV occurred in all groups: 27/45 (60\%) of MSA, $12 / 31(38.7 \%)$ of PD, and $4 / 14(28.6 \%)$ of PSP patients. During tilt table testing, mean arterial pressure decreased significantly in MSA and PD, but not PSP. A pathologic tilt table response was seen in all groups; (28/47) $59.6 \%$ of MSA, (17/33) $51.5 \%$ of $\mathrm{PD}$, and (2/15) $13.3 \%$ of PSP patients.

Cardiac sympathetic innervation assessed using Iodine-123 meta-iodobenzylguanidine (MIBG) revealed that the heart to mediastinum average count ratio for both early and late images was significantly lower in PD compared to normal controls, MSA, and PSP. ${ }^{31}$ These results indicate that patients with PD have an abnormality of cardiac sympathetic function.

Autonomic testing (Quantitative Sudomotor Axon Reflex Test; tilt table testing; heart rate response to deep breathing; Valsalva ratio; beat to beat BP during Valsalva, tilt, deep breathing) in 20/124 patients diagnosed with dementia with Lewy bodies referred for autonomic testing was compared to 20 patients with PD and 20 with MSA. ${ }^{29}$ Using the Composite Autonomic Severity Score (CASS), patients with DLB had dysautonomia intermediate between MSA and PD.

Using motor clinical criteria, 47 patients referred for autonomic testing with diagnoses of $\mathrm{PD}$, parkin- sonism, and MSA were classified as having probable $\mathrm{PD}(\mathrm{n}=19)$, probable MSA $(\mathrm{n}=14)$, and uncertain diagnosis $(\mathrm{n}=14) .{ }^{26}$ Subsequent results of formal autonomic testing (deep breathing, Valsalva, tilt table, sudomotor axon reflex, sweat test) failed to distinguish between probable MSA and probable PD patients.

One electromyography study demonstrated that urethral sphincter motor units in MSA are of greater duration than in $\mathrm{PD}$ and that the percent of abnormal motor units is greater than in PD (table E-3). ${ }^{22}$ Using a cutoff of $>20 \%$ motor unit prolongation, urethral sphincter EMG had a sensitivity of $62 \%$ and a specificity of $92 \%$ for predicting probable MSA. If the cutoff for the percent of abnormal units was set lower than $20 \%$, the gain in sensitivity led to overlap with $\mathrm{PD}$, decreasing specificity.

In a blinded study of anal sphincter EMG, no significant differences were observed between $\mathrm{PD}$ and MSA. ${ }^{23}$ In another study, anal sphincter EMG could distinguish MSA from normal controls, but not from patients with $\mathrm{PD}^{25}$ (table E-3).

Diagnostic neuroimaging.

MRI. Two Class III articles evaluated the ability of MRI to differentiate PD from MSA (table E-4). ${ }^{32,33}$ In the first study, 27 patients with $\mathrm{PD}$ and 24 patients with MSA had an MRI $1.5 \mathrm{~T}$ upon presentation. ${ }^{32}$ Diagnoses were confirmed clinically at least 1.5 years later. Putaminal hypointensity on T2weighted images was seen in $21 / 24$ patients with MSA (87.5\% sensitivity) compared to only $3 / 27$ patients with PD (88\% specificity). Putaminal hyperintensity on proton density weighted images was seen in 20/24 MSA patients compared to none of the PD patients (100\% specificity). Fast spin echo (FSE) protocol images had putaminal abnormalities in 9/20 MSA patients (45\% sensitivity) and none of the PD patients. The second study evaluated diffusionweighted MRI in $11 \mathrm{PD}$ patients, 10 MSA patients, and 7 healthy controls. ${ }^{33}$ Clinical diagnosis was made based on published criteria. Putaminal regional apparent diffusion coefficients were higher in MSA (median $0.791 \times 10^{3} / \mathrm{mm}^{2} /$ second) patients than $\mathrm{PD}$ patients $\left(0.698 \times 10^{3} / \mathrm{mm}^{2} /\right.$ second $)(p<0.001)$ or healthy volunteers $\left(0.727 \times 10^{3} / \mathrm{mm}^{2} /\right.$ second $)(p<$ 0.001).

Sonography. One Class III study compared brain parenchyma sonography in 25 patients with atypical parkinsonian syndromes (16 with MSA and 9 with PSP) to 25 patients with PD (table E-4). ${ }^{34}$ Diagnosis was made based on clinical criteria. Ninety-six percent $(24 / 25)$ of the PD patients had hyperechogenicity of the substantia nigra compared to only $2 / 23$ (9\%) of the patients with other parkinsonian syndromes $(p<0.001)$.

SPECT. Using clinical evaluation as the gold standard, five class III studies ${ }^{35-39}$ demonstrated that IBZM or B-CIT SPECT had $8 \%$ to $100 \%$ specificity in identifying clinically diagnosed PD patients, as compared to other parkinsonian syndromes. Sensitivity varied from 30 to $100 \%$ (table E-4).

PET. One Class III article reported the results of 
quantitative ${ }^{18} \mathrm{~F}$ Fluorodeoxyglucose (FDG) PET in 48 patients with atypical parkinsonian syndromes and 56 patients with likely PD (table E-4). ${ }^{40}$ Both groups were diagnosed based on clinical criteria. A linear combination of regional metabolic data of the caudate, lentiform, and thalamic values distinguished atypical parkinsonism from $\operatorname{PD}(p<0.0001)$. No studies using fluoro-dopa PET fulfilled inclusion criteria.

Conclusions. Falls at presentation or early in the disease course, poor response to levodopa, symmetry of motor signs, rapid progression (to Hoehn and Yahr stage 3 in 3 years), lack of tremor, and early dysautonomia (urinary urge incontinence, fecal incontinence, urinary retention requiring catheterization, persistent erectile failure, and symptomatic orthostatic hypotension) are signs that are probably useful in identifying patients with forms of parkinsonism other than PD in patients with mild disease (three Class II and one Class III studies). The signs are particularly useful when several are present.

Levodopa and apomorphine challenge tests are probably useful in distinguishing PD from other parkinsonian syndromes (one Class I and one Class II studies). The diagnostic yields appear to be comparable between the two tests. However, using clinical diagnosis as the gold standard, at least $30 \%$ of patients with PD will not be diagnosed by either test (false negative) and 20 to $30 \%$ of patients ultimately diagnosed with other forms of parkinsonism will have a positive test (false positive). The acute levodopa challenge appears to be a reliable predictor of the chronic response to levodopa. Pretreatment with domperidone in drug naïve patients is recommended to prevent peripheral side effects.

GH stimulation by clonidine may not be useful to distinguish PD from MSA (one Class II study).

Olfaction testing, using either the UPSIT or "Sniffin' Sticks," is probably useful in differentiating PSP and CBD from PD (three Class II studies). Although PD patients have decreased smell as compared to MSA, these differences are not as pronounced. Thus, a significant loss of smell is suggestive of PD rather than other parkinsonian syndromes.

Formal eye movement recordings may not be useful to distinguish PD from other forms of parkinsonism, due to significant overlap in the abnormalities observed (two Class III studies).

There is insufficient evidence to determine if urodynamic testing or urethral or anal sphincter EMG are useful in distinguishing PD from MSA (one Class III study for each modality).

Testing of autonomic function may not be useful to distinguish PD from other forms of parkinsonism (four Class III studies).

There is insufficient evidence to determine if iodine-123 meta-iodobenzylguanidine cardiac imaging is useful in differentiating PD from MSA or PSP (one Class III study).

MRI is possibly useful in distinguishing PD from
MSA (two Class III studies using different MRI techniques).

There is insufficient evidence to determine if brain parenchyma sonography is useful in distinguishing PD from atypical parkinsonian syndromes (one Class III study).

$\beta$-CIT and IBZM SPECT are possibly useful in distinguishing PD from ET and subjects (five Class III studies). There is insufficient evidence to determine if these modalities are useful in distinguishing PD from other forms of parkinsonism.

There is insufficient evidence to determine if FDG PET is useful for diagnosing PD (one Class III study).

Recommendations. Determining the presence of the following clinical features in early stages of disease should be considered to distinguish PD from other parkinsonian syndromes: 1 ) falls at presentation and early in the disease course, 2) poor response to levodopa, 3) symmetry at onset, 4) rapid progression (to Hoehn and Yahr stage 3 in 3 years), 5) lack of tremor, and 6) dysautonomia (urinary urgency/ incontinence and fecal incontinence, urinary retention requiring catheterization, persistent erectile failure, or symptomatic orthostatic hypotension) (Level B) (appendix E-4).

Levodopa and apomorphine challenge should be considered for confirmation when the diagnosis of $\mathrm{PD}$ is in doubt (Level B).

Olfaction testing should be considered to differentiate PD from PSP and CBD, but not PD from MSA (Level B).

There is insufficient evidence to determine whether levodopa and apomorphine challenge or olfaction testing have any advantage over the clinical diagnostic criteria of PD (Level U). Additionally, there is insufficient evidence to determine the optimal combination or sequence of these tests (Level U).

The following may not be useful in differentiating PD from other parkinsonian syndromes: GH stimulation with clonidine, electrooculography, and SPECT scanning (Level C).

There is insufficient evidence to support or refute the following as a means of distinguishing PD from other parkinsonian syndromes: urodynamics, autonomic testing, urethral or anal EMG, MRI, brain parenchyma sonography, and FDG PET (Level U).

Question 2: Which clinical features predict rate of disease progression? The ability to predict rate of progression of disease can benefit planning for long term patient care as well as permit the development of neuroprotective strategies. We identified seven studies (six Class II, one Class III) that evaluated whether factors such as age at disease onset, sex, and cognitive and motor symptoms can predict the progression of PD (table E-5). ${ }^{41-47}$

One Class II, single blind, retrospective, matched double cohort study found that PD patients with an older age at onset $(\geq 78)$ had greater motor impairment than patients with middle age onset (43 to 66) after a comparable disease duration of approxi- 
mately 5 years (33.3 vs 21.2 on total UPDRS; $p<$ 0.001). ${ }^{42}$ The group with older age at onset had more rigidity ( 5.2 vs $4.3, p=0.03$ ), bradykinesia ( $13 \mathrm{vs}$ $9.6, p=0.001)$, and axial impairment (12.8 vs 5.2 , $p<0.001$ ) than their younger onset counterparts as measured by UPDRS motor scores. Comorbidities including stroke, auditory deficits, and visual impairments were also more common in PD patients with an older age at onset. The increase in motor impairment in the older onset group was still evident after adjusting for comorbidities.

A single blind, Class II, retrospective cohort survey followed 297 consecutive $\mathrm{PD}$ patients at various stages of disease for at least 3 years (mean 6.4) and determined the rate of $\mathrm{PD}$ progression by the percent change in yearly total UPDRS scores. ${ }^{44}$ Patients were categorized as having either tremor-dominant or postural instability/gait difficulty (PIGD) dominant PD. Patients with an older age at onset $(>57$ years) had more progression in freezing and in parts I and II of the UPDRS score (mentation and activities of daily living) compared with patients with middle age onset disease. Rate of progression as measured by the total UPDRS was greater in the group with age at onset $>57$ years as compared to the group with age at onset $<57$ years (slopes 2.65 vs 0.89 ). The slopes (annual rate of decline) in UPDRS scores were steeper for the PIGD group compared to the tremor-dominant group when adjusted for age at the initial visit (slopes 2.82 vs 1.59 ). UPDRS parts I and II subscores progressed more quickly in men than women.

A Class II, single blind, retrospective cohort study demonstrated that $\mathrm{PD}$ patients with rigidity/hypokinesia as a first symptom reached Hoehn and Yahr stage III sooner than patients who experienced tremor alone at disease onset $(p=0.001){ }^{47}$ Patients with tremor at disease onset developed dementia less frequently $(p=0.05)$ and later $(p=0.001)$ than patients with rigidity/hypokinesia at onset $(0=0.05)$.

A Class II, single blind, historical cohort study of $89 \mathrm{PD}$ patients examined predictors of nursing home placement and survival. ${ }^{46}$ Predictors included age at onset of $\mathrm{PD}$, dementia, and responsiveness to levodopa. Early nursing home placement was associated with older age at onset (RR 3.1), presence of dementia (RR 2.3), and unresponsiveness to dopaminergics (RR 2.0). Longer survival was associated with dopaminergic responsiveness (relative risk of death 0.4 ), absence of dementia (RR 0.4), and younger age at onset (RR 0.3). Multivariate analysis revealed that older age at onset was the sole independent predictor of both nursing home placement and death.

A Class II, single blind, prospective cohort survey examined whether clinical characteristics could predict cognitive decline. ${ }^{43} \mathrm{~A}$ total of $104 \mathrm{PD}$ patients at various stages of disease and 60 healthy volunteers of comparable age and education underwent 14 tests of memory, language, frontal lobe, and visuospatial capacity. Eighty percent of participants had more than one test session, and the repeat testing was completed over a period of 1 to 10 years (mean 3.6 years). A delay in cognitive decline was associated with a younger age at onset of PD. Severity of motor impairment, as measured by the Hoehn and Yahr scale, was related to impairment on almost all cognitive tests. Once cognitive decline began, it occurred at a similar rate in all age groups.

One Class II prospective cohort study evaluated the progression of PD in 34 patients using levodopa half-life kinematic-dynamic modeling in an attempt to assess residual neuronal function. ${ }^{41}$ The study proposed that a reduction in levodopa half-life with disease progression could signify abnormal nigrostriatal turnover, possibly due to the loss of dopamine storage capacity. Levodopa half-life values correlated negatively with the severity of symptoms ( $\mathrm{r}=$ $-0.652, p<0.0001$ ) and progressively decreased along with the patients' clinical stage (annual reduction of 37 minutes in $\mathrm{H} \& \mathrm{Y} 1$ vs 6.5 minutes in $\mathrm{H} \& \mathrm{Y}$ III). PD patients without tremor had a larger annual reduction in levodopa half-life compared to patients with tremor, 28 minutes vs 11 minutes.

A Class III, case control study using a subset of the DATATOP cohort found that patients with early onset of disease (first symptom at $\leq 40$ years) had a slower progression of disease and better cognitive function than patients with late onset symptoms $(\geq 70) .{ }^{45}$ Early onset patients reached the same level of disability in 2.9 years, vs 1.7 years for late onset patients. Patients with late onset disease were also more occupationally disabled than their early onset counterparts. Patients with PIGD had a more rapid rate of disease progression and greater subjective, intellectual, motor, and occupational impairment than tremor dominant patients. Patients in the benign group of PD (duration of PD symptoms at least 4 years before study entry) had an earlier age at onset (55.4 years) compared to the malignant group (68.2 years). The malignant group was defined as PD patients with symptoms for $\leq 1$ year who progressed during this period of time to a stage of 2.5 on the Hoehn and Yahr scale. Tremor was the initial disease symptom in $74 \%$ of patients in the benign group compared with $55 \%$ of patients in the malignant group. Although not significant, a trend was suggested when adjusted for age $(p=0.06)$.

Conclusions. Older age at onset (variably defined as over age 57 to 78 years) (two Class II and one Class III studies) and rigidity/hypokinesia as a presenting symptom (two Class II studies) are factors which are probably useful in predicting a more rapid rate of motor progression of PD.

The presence of associated comorbidities (one Class II study), features of PIGD (one Class II and one Class III studies), and male sex (one Class II study) are factors that are possibly useful for predicting a more rapid rate of motor progression of PD.

Tremor as the initial presentation is a factor that is possibly useful in predicting slower progression and a longer response to levodopa therapy (one Class II and one Class III studies). 
Older age at onset and initial manifestations of hypokinesia/rigidity are factors that are probably useful in predicting earlier development of cognitive decline and dementia (two Class II and one Class III studies).

Older age at onset, dementia, and decreased dopamine responsiveness are factors that are possibly useful in predicting an increased risk for nursing home placement and shorter survival after diagnosis (one Class II study).

Recommendations. In patients with newly diagnosed PD, older age at onset and rigidity/hypokinesia as an initial symptom should be used to predict more rapid rate of motor progression (Level B).

The presence of associated comorbidities (stroke, auditory deficits, and visual impairments), PIGD, and male sex may be used to predict faster rate of motor progression (Level C).

Tremor as a presenting symptom may be used to predict a more benign course and longer therapeutic benefit to levodopa (Level C).

Older age at onset and initial hypokinesia/rigidity should be used to predict earlier development of cognitive decline and dementia (Level B).

Older age at onset, dementia, and decreased dopamine responsiveness may be used to predict earlier nursing home placement as well as decreased survival (Level C).

Recommendations for future research. Al-
though $\mathrm{PD}$ is a common disorder, accurate diagnosis remains a challenge in early stages of the disease. Clinical examination with long-term follow-up appears to be the best method for confirmation of diagnosis during the patient's lifetime. Further studies are needed to identify other techniques, such as neuroimaging and levodopa challenge tests, that may improve diagnostic accuracy and adequately address disease progression, and to determine superiority to the clinical examination.

In the future, there may be an increasing role for genetic testing to make the diagnosis of PD. However, the development of any new diagnostic test (e.g., neuroimaging, genetic screening) will require long-term follow-up and autopsy confirmation to determine its accuracy. Methods for presymptomatic testing to identify patients who are at risk of developing PD are also critical, particularly for testing of neuroprotective strategies. Similarly, knowledge of disease progression will play a key role, not only in providing useful clinical information, but in assessing the benefit of neuroprotective interventions. Funding agencies need to recognize the necessity of supporting long term studies to answer these questions.

Disclaimer. This statement is provided as an educational service of the American Academy of Neurology. It is based on an assessment of current scientific and clinical information. It is not intended to include all possible proper methods of care for a particular neurologic problem or all legitimate criteria for choosing to use a specific procedure. Neither is it intended to exclude any reasonable alternative methodologies. The AAN recognizes that specific patient care decisions are the prerogative of the patient and the physician caring for the patient, based on all of the circumstances involved.

Disclosure. Dr. Suchowersky has received consulting fees from Teva, speaker fees from GlaxoSmithKline, and research funds from Boehringer Ingelheim, Kyowa, Merck, Amarin, Cephalon, Swartz-Pharma, and Solstice Neuroscience. Dr. Reich has received research funds from Guilford Pharmaceuticals and Cephalon. Dr. Perlmutter has received unrestricted educational funds from Medtronic. Dr. Zesiewicz has received consulting fees from UCB Pharma and Schwartz Pharma, speaker fees from Boehringer Ingelheim, GlaxoSmithKline, Novartis and Medtronic, and research funds from Boehringer Ingelheim, GlaxoSmithKline, Novartis and Merck. Dr. Weiner is a consultant for Teva, a speaker for Boehringer Ingelheim, and has received research funds from Boehringer Ingelheim and Teva. Dr. Gronseth has nothing to disclose.

\section{Acknowledgment}

The authors thank Wendy Edlund and Nancy King for administrative support and Andrew Wilner, MD, for assistance in manuipt preparation.

\section{References}

1. Strickland D, Bertoni JM. Parkinson's prevalence estimated by a state registry. Mov Disord 2004;19:318-323.

2. Hughes AJ, Daniel SE, Kilford L, Lees AJ. Accuracy of clinical diagnosis of idiopathic Parkinson's disease: a clinico-pathological study of 100 cases. J Neurol Neurosurg Psychiatry 1992;55:181-184.

3. Gelb DJ, Oliver E, Gilman S. Diagnostic criteria for Parkinson disease. Arch Neurol 1999;56:33-39.

4. Hughes AJ, Daniel SE, Lees AJ. Improved accuracy of clinical diagnosis of Lewy body Parkinson's disease. Neurology 2001;57:1497-1499.

5. Hughes AJ, Ben-Shlomo Y, Daniel SE, Lees AJ. What features improve the accuracy of clinical diagnosis in Parkinson's disease: a clinicopathologic study. Neurology 1992;42:1142-1146.

6. Litvan I, Bhatia KP, Burn DJ, et al. Movement Disorders Society Scientific Issues Committee report: SIC Task Force appraisal of clinical diagnostic criteria for Parkinsonian disorders. Mov Disord 2003;18:467-486.

7. Colosimo C, Albanese A, Hughes AJ, de Bruin VM, Lees AJ. Some specific clinical features differentiate multiple system atrophy (striatonigral variety) from Parkinson's disease. Arch Neurol 1995;52:294298.

8. Jankovic J, Rajput AH, McDermott MP, Perl DP. The evolution of diagnosis in early Parkinson disease. Parkinson Study Group. Arch Neurol 2000;57:369-372.

9. Wenning GK, Ebersbach G, Verny M, et al. Progression of falls in postmortem-confirmed parkinsonian disorders. Mov Disord 1999;14: 947-950.

10. Wenning GK, Ben-Shlomo Y, Hughes A, Daniel SE, Lees A, Quinn NP. What clinical features are most useful to distinguish definite multiple system atrophy from Parkinson's disease? J Neurol Neurosurg Psychiatry 2000;68:434-440.

11. Albanese A, Bonuccelli U, Brefel C, et al. Consensus statement on the role of acute dopaminergic challenge in Parkinson's disease. Mov Disord 2001;16:197-201.

12. Hughes AJ. Apomorphine test in the assessment of parkinsonian patients: a meta-analysis. Adv Neurol 1999;80:363-368.

13. Clarke CE, Davies P. Systematic review of acute levodopa and apomorphine challenge tests in the diagnosis of idiopathic Parkinson's disease. J Neurol Neurosurg Psychiatry 2000;69:590-594.

14. Merello M, Nouzeilles MI, Arce GP, Leiguarda R. Accuracy of acute levodopa challenge for clinical prediction of sustained long-term levodopa response as a major criterion for idiopathic Parkinson's disease diagnosis. Mov Disord 2002;17:795-798. 
15. Rossi P, Colosimo C, Moro E, Tonali P, Albanese A. Acute challenge with apomorphine and levodopa in Parkinsonism. Eur Neurol 2000;43: 95-101.

16. Strijks E, van't Hof M, Sweep F, Lenders JW, Oyen WJ, Horstink MW. Stimulation of growth-hormone release with clonidine does not distinguish individual cases of idiopathic Parkinson's disease from those with striatonigral degeneration. J Neurol 2002;249:1206-1210.

17. Ward CD, Hess WA, Calne DB. Olfactory impairment in Parkinson's disease. Neurology 1983;33:943-946.

18. Doty RL, Golbe LI, McKeown DA, Stern MB, Lehrach CM, Crawford D. Olfactory testing differentiates between progressive supranuclear palsy and idiopathic Parkinson's disease. Neurology 1993;43:962-965.

19. Muller A, Reichmann H, Livermore A, Hummel T. Olfactory function in idiopathic Parkinson's disease (IPD): results from cross-sectional studies in IPD patients and long-term follow-up of de-novo IPD patients. J Neural Transm 2002;109:805-811.

20. Wenning GK, Shephard B, Hawkes C, Petruckevitch A, Lees A, Quinn N. Olfactory function in atypical parkinsonian syndromes. Acta Neurol Scand 1995;91:247-250.

21. Bordet R, Benhadjali J, Destee A, Hurtevent JF, Bourriez JL, Guieu JD. Sympathetic skin response and $R-R$ interval variability in multiple system atrophy and idiopathic Parkinson's disease. Mov Disord 1996; 11:268-272.

22. Eardley I, Quinn NP, Fowler CJ, et al. The value of urethral sphincter electromyography in the differential diagnosis of parkinsonism. $\mathrm{Br}$ J Urol 1989;64:360-362.

23. Giladi N, Simon ES, Korczyn AD, et al. Anal sphincter EMG does not distinguish between multiple system atrophy and Parkinson's disease. Muscle Nerve 2000;23:731-734.

24. Holmberg B, Kallio M, Johnels B, Elam M. Cardiovascular reflex testing contributes to clinical evaluation and differential diagnosis of Parkinsonian syndromes. Mov Disord 2001;16:217-225.

25. Libelius R, Johansson F. Quantitative electromyography of the external anal sphincter in Parkinson's disease and multiple system atrophy. Muscle Nerve 2000;23:1250-1256.

26. Riley DE, Chelimsky TC. Autonomic nervous system testing may not distinguish multiple system atrophy from Parkinson's disease. J Neurol Neurosurg Psychiatry 2003;74:56-60.

27. Rascol O, Sabatini U, Simonetta-Moreau M, Montastruc JL, Rascol A Clanet M. Square wave jerks in parkinsonian syndromes. J Neurol Neurosurg Psychiatry 1991;54:599-602.

28. Stocchi F, Barbato L, Carbone A, et al. Urodynamic study in the differential diagnosis between multiple system atrophy and Parkinson's disease. Adv Neurol 1993;60:434-437.

29. Thaisetthawatkul P, Boeve BF, Benarroch EE, et al. Autonomic dys function in dementia with Lewy bodies. Neurology 2004;62:1804-1809.

30. Vidailhet M, Rivaud S, Gouider-Khouja N, et al. Eye movements in parkinsonian syndromes. Ann Neurol 1994;35:420-426.

31. Yoshita M. Differentiation of idiopathic Parkinson's disease from stria tonigral degeneration and progressive supranuclear palsy using iodine-
123 meta-iodobenzylguanidine myocardial scintigraphy. J Neurol Sci 1998;155:60-67.

32. Righini A, Antonini A, Ferrarini M, et al. Thin section MR study of the basal ganglia in the differential diagnosis between striatonigral degeneration and Parkinson disease. J Comput Assist Tomogr 2002;26:266-271.

33. Schocke MF, Seppi K, Esterhammer R, et al. Diffusion-weighted MRI differentiates the Parkinson variant of multiple system atrophy from PD. Neurology 2002;58:575-580.

34. Walter U, Niehaus L, Probst T, Benecke R, Meyer BU, Dressler D. Brain parenchyma sonography discriminates Parkinson's disease and atypical parkinsonian syndromes. Neurology 2003;60:74-77.

35. Gerschlager W, Bencsits G, Pirker W, et al. [123I]beta-CIT SPECT distinguishes vascular parkinsonism from Parkinson's disease. Mov Disord 2002;17:518-523.

36. Kim YJ, Ichise M, Ballinger JR, et al. Combination of dopamine transporter and D2 receptor SPECT in the diagnostic evaluation of PD, MSA, and PSP. Mov Disord 2002;17:303-312.

37. Asenbaum S, Pirker W, Angelberger P, Bencsits G, Pruckmayer M, Brucke T. [123I]beta-CIT and SPECT in essential tremor and Parkinson's disease. J Neural Transm 1998;105:1213-1228.

38. Messa C, Volonte MA, Fazio F, et al. Differential distribution of striatal [123I]beta-CIT in Parkinson's disease and progressive supranuclear palsy, evaluated with single-photon emission tomography. Eur J Nucl Med 1998;25:1270-1276.

39. Varrone A, Marek KL, Jennings D, Innis RB, Seibyl JP. [(123)I]betaCIT SPECT imaging demonstrates reduced density of striatal dopamine transporters in Parkinson's disease and multiple system atrophy. Mov Disord 2001;16:1023-1032.

40. Antonini A, Kazumata K, Feigin A, et al. Differential diagnosis of parkinsonism with [18F]fluorodeoxyglucose and PET. Mov Disord 1998; 13:268-274.

41. Contin M, Riva R, Martinelli P, Cortelli P, Albani F, Baruzzi A. A levodopa kinetic-dynamic study of the rate of progression in Parkinson's disease. Neurology 1998;51:1075-1080.

42. Diederich NJ, Moore CG, Leurgans SE, Chmura TA, Goetz CG. Parkinson disease with old-age onset: a comparative study with subjects with middle-age onset. Arch Neurol 2003;60:529-533.

43. Locascio JJ, Corkin S, Growdon JH. Relation between clinical characristics of Parkinson's disease and cognitive decline. J Clin Exp Neurosychol 2003,25:94-109.

44. Jankovic J, Kapadia AS. Functional decline in Parkinson disease. Arch Neurol 2001;58:1611-1615.

Jankovic J, McDermott M, Carter J, et al. Variable expression of Parkinson's disease: a base-line analysis of the DATATOP cohort. The Parkinson Study Group. Neurology 1990;40:1529-1534.

46. Parashos SA, Maraganore DM, O'Brien PC, Rocca WA. Medical services utilization and prognosis in Parkinson disease: a population-based study. Mayo Clin Proc 2002;77:918-925.

47. Roos RA, Jongen JC, van der Velde EA. Clinical course of patients with idiopathic Parkinson's disease. Mov Disord 1996;11:236-242. 


\section{Neurology}

\section{Practice Parameter: Diagnosis and prognosis of new onset Parkinson disease (an evidence-based review) [RETIRED]: Report of the Quality Standards Subcommittee of the American Academy of Neurology}

O. Suchowersky, S. Reich, J. Perlmutter, et al.

Neurology 2006;66;968-975 Published Online before print April 2, 2006

DOI 10.1212/01.wnl.0000215437.80053.d0

\section{This information is current as of April 2, 2006}

\section{Updated Information \& Services}

Supplementary Material

\section{References}

Citations

Permissions \& Licensing

Reprints including high resolution figures, can be found at: http://n.neurology.org/content/66/7/968.full

Supplementary material can be found at: http://n.neurology.org/content/suppl/2006/04/06/66.7.968.DC1 http://n.neurology.org/content/suppl/2006/04/03/01.wnl.0000215437.8 0053.d0.DC1

This article cites 47 articles, 14 of which you can access for free at: http://n.neurology.org/content/66/7/968.full\#ref-list-1

This article has been cited by 14 HighWire-hosted articles: http://n.neurology.org/content/66/7/968.full\#\#otherarticles

Information about reproducing this article in parts (figures,tables) or in its entirety can be found online at:

http://www.neurology.org/about/about_the_journal\#permissions

Information about ordering reprints can be found online: http://n.neurology.org/subscribers/advertise

Neurology ${ }^{\circledR}$ is the official journal of the American Academy of Neurology. Published continuously since 1951, it is now a weekly with 48 issues per year. Copyright . All rights reserved. Print ISSN: 0028-3878. Online ISSN: 1526-632X.

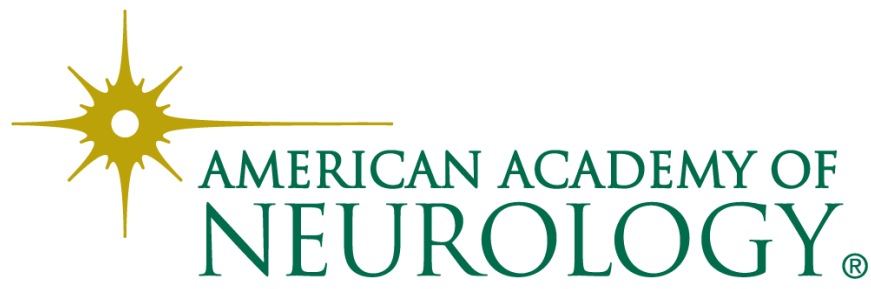

